\title{
Inequivalent Quantizations of Gauge Theories
}

\author{
Kenichi Horie团 \\ Institut für Physik der Johannes-Gutenberg-Universität, \\ 55099 Mainz, Germany
}

January 5, 2018

\begin{abstract}
It is known that the quantization of a system defined on a topologically non-trivial configuration space is ambiguous in that many inequivalent quantum systems are possible. This is the case for multiply connected spaces as well as for coset spaces. Recently, a new framework for these inequivalent quantizations approach has been proposed by McMullan and Tsutsui, which is based on a generalized Dirac approach. We employ this framework for the quantization of the Yang-Mills theory in the simplest fashion. The resulting inequivalent quantum systems are labelled by quantized non-dynamical topological charges.
\end{abstract}

Number of pages: 24

${ }^{*}$ Present address: High Energy Accelerator Research Organization (KEK), Tanashi Branch, Tokyo 188-8501, Japan 


\section{Introduction}

In gauge theory, the highly non-trivial topology of the underlying gauge orbit space directly leads to or is related to many physically interesting phenomena. Some of the major examples are the $\theta$-vacua, topological terms like Chern-Simons term or Pontrjagin term, and also chiral anomaly, see e.g. Refs. 1, 2, 3, and 4. The important point to note is that these facets are not consequences of the non-trivial topology alone but arise only upon quantization or, to be more distinct, upon reducing the simple quantum system on the total gauge potential space, which is affine, to the complicated gauge orbit space by dividing out the gauge group. Thus, in view of the prominent status of the gauge theories in physics, the quantization and reduction procedures of these systems must be investigated with ever more effort.

In this short note we make first steps in applying the framework of inequivalent quantizations proposed recently $y^{5,6}$ to gauge theories and hint at further possible extensions.

Many papers have appeared dealing with the methods of quantization on a nontrivial (finite dimensional) configuration space other than the well known case of linear space, see e.g. Refs. 5, 7, 8, 9, 10, and 11 and references therein as well as numerous other contributions. These works are of physical interest in their own rights, but the generalized Dirac approach of McMullan and Tsutsui ${ }^{5}$ based on the work of Mackey ${ }^{7}$ is directly applicable to gauge theory. To understand the ideas behind their approach, consider a classical system with first class constraints

$$
\phi_{i}=0
$$

which, by definition, build up a closed algebra under the Poisson brackets. Due to Dirac $^{12}$ the quantization of such a constrained system may be performed by first 
quantizing the system on the entire phase space without the constraints and then imposing the constraints (1) afterwards as operator conditions on quantum states,

$$
\hat{\phi}_{i}|\psi\rangle=0
$$

On the other hand, Mackey's approach to quantization on coset spaces takes a different path. The classical system is quantized directly on the reduced space $G / H$ by generalizing the canonical commutation relations and using irreducible representations of what he called the imprimitivity relations. ${ }^{7}$ We will not go into details of this somewhat abstract algebraic procedure, but remark that Mackey's work was one of the first to show the existence of inequivalent quantum sectors on the coset space. In Ref. 5 this algebraic procedure was translated into the commonly used framework of Dirac formalism, thus, as we will see, making possible the application of inequivalent quantizations also to Yang-Mills theory. The generalization capable of incorporating the inequivalent quantizations within Dirac's approach consists in replacing the constraints (国) by

$$
\phi_{i}-K_{i}=0
$$

The constraints (3) define different classical systems which in case of coset space $G / H$ correspond to different foliations of the Marsden-Weinstein reduction. ${ }^{13}$ However, upon quantization the arbitrary numbers $K_{i}$ turn out to be discrete multiples of $\hbar$ and label the inequivalent quantum superselection sectors. ${ }^{14}$ This implies the important fact that in the classical limit $\hbar \longrightarrow 0$ all these inequivalent quantum systems reduce to the unique classical system described by (1) and not to those classically different systems described by (3). From standard viewpoint quantum physical systems play a more fundamental role in nature than the corresponding 
classical systems in that nature is described ultimatively by quantum rather than classical physics. Accordingly, regardless of actually how the quantum sectors have been derived, solely due to the fact that all these quantum theories have a unique classical limit, we may talk about 'inequivalent quantizations' of this particular classical system, even though these quantum sectors have been obtained by quantizing different classical systems. These inequivalent quantum sectors are all legitimate physical systems, whereas among the classically inequivalent systems there is one distinguished system (11) to which all quantum systems reduce to in the classical limit. The constraints (3) are now partially second class ('anomalous') and care must be taken when quantizing. The resulting quantum theories are equipped with an induced connection ${ }^{10}$ and a generalized spin, i.e. the equations of motion on the coset space describe a particle with certain spin degrees of freedom depending on $K_{i}$ and minimally coupled to an external Yang-Mills type connection.

One of the most prominent constrained physical system is gauge theory, where the true configuration space is obtained as the quotient of the affine gauge potential space by the gauge group. This structure is similar to the coset space structure considered in Refs. 5 and 14, and it is interesting to know something about the possible inequivalent quantum systems which in the classical limit reduce to the given classical gauge theory. One of the questions we address in this paper is the physical role of the possible parameter which labels the quantum sectors.

When this method of inequivalent quantizations is applied to Yang-Mills theory, the new constraints (3) turn out to correspond to modified Gauss laws in general, see in this context also Refs. 15 and 16. However, contrary to the case of homogeneous spaces, a number of legitimate modifications are possible, which are in general field dependent as anticipated in Ref. 5. Among the possible modifications we pick up 
the most "simple" one. As a result, the modified Gauss laws induce non-dynamical charges. Upon quantization of the Yang-Mills theory these charges become quantized and describe topologically distinct field configurations.

The organization of this paper is as follows. In order to apply these ideas to YangMills theory we must be aware that the relevant configuration space is no longer a coset of the type $G / H$ but the base space $\mathcal{C} / \mathcal{G}$ of a principal bundle, where $\mathcal{C}$ is the total space of connections, and the structure group is given by the gauge group $\mathcal{G}$. (More precisely, we must restrict $\mathcal{C}$ to the dense set of irreducible connections since otherwise the group action is non-free.) Thus, as briefly mentioned in Ref. 6, the generalized Dirac quantization must be set up for principal bundles, which is the main task of the next section. In the third section, the geometric structures on the gauge orbit space are clarified and compared to the finite dimensional case, upon which the actual quantization is carried out. The last section discusses the results and hints at some further developments.

\section{Particle on a Principal Bundle}

In this section we extract from Refs. 5 and 6 the mathematical structures essential for the inequivalent quantizations of Yang-Mills system and, in order to adapt the geometrical setting, consider a classical point particle moving on a principal bundle.

Although such a physical system is already known in literature, ${ }^{11,13,17,18}$ the following presentation differs from what have been appeared hitherto in that it is kept as simple as possible and, furthermore, will allow a direct conversion to the YangMills system in the next section.

Let $G$ be a semisimple Lie group, such that its Lie algebra, denoted by $\mathcal{L}(G)$, 
possesses a non-degenerate metric $\operatorname{Tr}(R S), R, S \in \mathcal{L}(G)$, defined by some multiple of the trace function in a certain irreducible matrix representation. Let $P$ be a principal bundle with this structure group $G$ over a basemanifold $B$ and consider the following $G$-invariant Lagrangian

$$
L=\frac{1}{2} g_{\mu \nu} \dot{u}^{\mu} \dot{u}^{\nu}-V(u)=\frac{1}{2} g(\dot{u}, \dot{u})-V(u),
$$

where $\dot{u} \in T_{u} P$ is the velocity of the particle, and $V(u)$ and $g$ are $G$-invariant potential and metric, respectively. Thus, $V(u)=V(u \Lambda)$ and $R_{\Lambda *} g=g$, where $\Lambda \in G$ and $R_{\Lambda *}$ denotes the pull-back defined by the right group action $R_{\Lambda}(u)=u \Lambda$. The Hamiltonian corresponding to (四) reads

$$
H=\frac{1}{2} g(p, p)+V(u),
$$

where $p$ is the particle momentum and $g$ in this equation stands for the inverse metric on the cotangent space $T_{u}^{*} P$. In what follows we identify the cotangent space with tangent space via the metric, and the mechanical system of the point particle has as its phase space the tangent bundle $T P$. This technical issue is not really essential but is introduced here to make the application of inequivalent quantizations to Yang-Mills theory in the next section more transparent.

Having fixed the phase space and the Hamiltonian we now define the symplectic structure on the tangent bundle $T P$. For this consider on the cotangent bundle the canonical 1-form $\Theta_{0}=p_{\mu} d u^{\mu}$ and the canonical symplectic structure $\Omega_{0}:=-d \Theta_{0}$ derived from it. We pull back this symplectic 2 -form $\Omega_{0}$ via the metric to the tangent bundle. Thus our symplectic 2-form reads $\Omega=-d \Theta$, where $\Theta$ is the pull-back of $\Theta_{0}$ given by

$$
\Theta=g_{\mu \nu} p^{\mu} d u^{\nu}
$$


Note that in this equation and in (5) the momentum is defined on the tangent space, $p=p^{\mu} \partial_{\mu}$

The $G$-symmetry of the system under consideration is expressed by the momentum maps $J$, see e.g. Ref. 19. These are functions on the phase space TP defined for each element $R \in \mathcal{L}(G)$ of the Lie algebra by

$$
J(R)(p):=g\left(p, R^{+}\right)
$$

where $R^{+}$denotes the fundamental vector field of $R$. These momenta are conserved under the Hamiltonian flow. Furthermore, for the special symplectic structure $\Omega$ at hand, $J$ is a homomorphism of the Lie algebra of $G$ to that of functions on TP under the Poisson bracket, ${ }^{19}$

$$
\{J(R), J(S)\}=J([R, S])
$$

Just as in the case of the symplectic structure $\Omega$, the momentum map $J$ (7) is the pull-back via the metric of a corresponding canonical momentum mapping ${ }^{19}$ on the cotangent bundle. Thus both $\Omega$ and $J$ depend on the $G$-invariant metric $g$. Now the metric $g$ also defines another geometric structure on $T P$, namely a $G$-connection. Let us note here the link between this derived connection and the momentum map. The connection is given by the decomposition of the tangent space $T_{u} P$ into the vertical component $V_{u} P$ along the fibres and the orthogonal horizontal complement $H_{u} P$. If $\left\{T_{a}\right\}$ denotes an orthonormal basis of $\mathcal{L}(G)$, then $V_{u} P$ is spanned by $T_{a}^{+}$, and the corresponding orthogonal projection of $T_{u} P$ onto $V_{u} P$ reads

$$
\begin{aligned}
\Pi: T_{u} P & \longrightarrow V_{u} P \\
p & \longmapsto g_{u}\left(p, T_{a}^{+}\right) \eta^{a b} T_{b}^{+} .
\end{aligned}
$$


The matrix $\eta^{a b}$ is the inverse of $\eta_{a b}:=g_{u}\left(T_{a}{ }^{+}, T_{b}{ }^{+}\right)$and is generally $u$-dependent. It is easy to show that $\Pi$ is independent of the basis chosen. Now it is not difficult to confirm that the $\mathcal{L}(G)$-valued connection 1 -form $\omega$ is obtained by simply replacing the fundamental vector field $T_{b}{ }^{+}$in (9) by its generator $T_{b}$,

$$
\omega(p)=g\left(p, T_{a}^{+}\right) \eta^{a b} T_{b}
$$

Thus we see that the momentum to each generator $T_{a}$,

$$
r_{a}:=J\left(T_{a}\right)
$$

is linked to the connection via

$$
r_{a}(p)=\operatorname{Tr}\left(\omega(p) T_{b}\right) \eta_{b a}
$$

Turning back to mechanics, to recover the dynamics of the particle on the base $B$, we will employ the Dirac constraints ${ }^{12}$ in accordance with Ref. 5 . If we introduce constraints

$$
r_{a}=0
$$

then from (8) we have

$$
\left\{r_{a}, r_{b}\right\}=f_{a b}^{c} r_{c}
$$

with structure constants $f_{a b} c$. Thus the $r_{a}$ build up a system of first class constraints consistent with the Hamiltonian, $\left\{r_{a}, H\right\}=0$, since momentum maps are conserved quantities.

However, and this is the salient point recovered in Ref. 5 from the analysis of Mackey's inequivalent quantizations procedure, there is no stringent reason to restrict ourselves to the constraints (13) but we are free to introduce (at the first 
place) an arbitrary element $K$ in the Lie algebra and replace (13) by

$$
r_{a}-\operatorname{Tr}\left(T_{a} K\right)=r_{a}-K_{a}=0
$$

which is formally the same as the modified constraints employed by McMullan and Tsutsui for the homogeneous space. The details of the physical motivation which led to this modified constraints may be found in Ref. 5 . Note that the reductions of the physical system via the modified constraints (15) corrospond to Marsden-Weinstein reductions. ${ }^{13,11,17,18}$

Let us remark that on a general principal bundle the modified constraints (15) are not the only possibility. In fact any modification will do, provided it reduces to the one considered in Ref. 5 for the homogeneous space. This restriction applies as long as we base our considerations on Mackey's work. Thus the full content of the idea of inequivalent quantizations is, insofar as its classical reduction part is considered, more general than the Marsden-Weinstein reduction considered above. Another natural modification of (13) matching this condition is $\left(p \in T_{u} P\right)$

$$
r_{a}(p)-g\left(T_{a}^{+}, K^{+}\right)=J\left(T_{a}\right)\left(p-K^{+}\right)=0 .
$$

The reason why we disregard this possibility is that the $K$-term is base point dependent in general and thus leads to a complication of the constraint algebra.

We shall now turn to the quantization of the so modified constrained system. The following discussion of quantization has been presented in Refs. 5 and 6 in great detail and is therefore kept brief.

The new constraints (15) are no more all first class, since the Poisson bracket of two of them can not be expressed with these constraints alone but acquires an additional term $\operatorname{Tr}\left[T_{a}, T_{b}\right] K$. This term vanishes if one of both generators lies in the 
kernel of the adjoint $\operatorname{map} \operatorname{ad}_{K}$. Thus (15) contains a first class subset given by

$$
\phi_{s}=J\left(T_{s}\right)-\operatorname{Tr} T_{s} K=r_{s}-K_{s}=0
$$

where $T_{s}$ are chosen to span $\operatorname{Ker}\left(\operatorname{ad}_{K}\right)=: s_{K}$, which decomposes as $s_{K}=t \oplus c$, where $t$ is the Cartan subalgebra containing $K$ and $c$ a possible orthogonal complement if $K$ is not regular semisimple. Let $S_{K}$ denote the Lie subgroup of $G$ generated by $s_{K}$.

Constraints other than (17) are second class, and for the constrained system there remains a gauge symmetry with respect to $S_{K}$-transformations. For these we introduce gauge fixing conditions by the functions

$$
\xi_{s}=\xi_{s}(u, p)=0
$$

The new system will be quantized by the path-integral method. In order to path integrate the Hamiltonian (5) over the phase space, we have to take into account both the first class constraints (17), the other second class constraints from (15), and also the above gauge fixings. Denoting all these different constraints by $\phi_{k}$ we obtain

$$
\begin{aligned}
Z & =\int \prod \Omega_{0}^{N} \delta\left(\phi_{k}\right) \operatorname{det}^{\frac{1}{2}}\left|\left\{\phi_{k}, \phi_{k^{\prime}}\right\}\right| \exp \left(\frac{i}{\hbar}\left(\int \Theta_{0}-H d t\right)\right) \\
& =\int \mathcal{D} p^{\mu} \mathcal{D} u^{\nu}\left(\prod \operatorname{det} g\right) \delta\left(\phi_{k}\right) \operatorname{det}^{\frac{1}{2}}\left|\left\{\phi_{k}, \phi_{k^{\prime}}\right\}\right| \exp \left(\frac{i}{\hbar}\left(\int d t g(p, \dot{u})-H\right)\right)
\end{aligned}
$$

The first path-integral (19) has been written on the cotangent bundle $T^{*} P$ using the product of the canonical Liouville form $\Omega_{0}^{N}$ over time $t$ and the canonical 1form $\Theta_{0}$. Note that the measure $\prod \Omega_{0}{ }^{N}$ is usually written more simply as $\mathcal{D} p_{\mu} \mathcal{D} u^{\nu}$, where $p_{\mu}$ and $u^{\nu}$ are canonical variables. The second expression (20) is defined on the tangent bundle $T P$, where the determinant of the metric comes in owing to the change of variable $p^{\mu}=g^{\mu \nu} p_{\mu}$. Note that $\Theta=g_{\mu \nu} p^{\mu} d u^{\nu}=g_{\mu \nu} p^{\mu} \dot{u}^{\nu} d t$. As 
explained in Ref. 5, by a certain choice of the form of the gauge fixing conditions (18), it is possible to integrate out the momentum. In so doing one can actually implement the constraints (15). Concerning this calculation we note briefly that it is done by decomposing the momentum $p$ and the velocity $\dot{u}$ via the projection (9) into the corresponding vertical and horizontal components, whereupon the vertical projection $\Pi(p)(9)$ of the momentum $p$ can be entirely replaced by an expression of $K$. Finally, the following path-integral over the configuration space is obtained

$$
Z=\int \mathcal{D} u \prod \operatorname{det} g \delta\left(\xi_{s}\right) \operatorname{det}\left|\left\{\phi_{s}, \xi_{s^{\prime}}\right\}\right| \exp \left(\frac{i}{\hbar} \int L_{\text {tot }} d t\right)
$$

where the total Lagrangian is a sum of the original Lagrangian (4) projected onto the base space $B$ and additional terms linear and quadratic in $K$,

$$
L_{\mathrm{tot}}=\frac{1}{2} g(\dot{v}, \dot{v})-V(u)+\operatorname{Tr}(K \omega(\dot{w}))-\frac{1}{2} K_{a} K_{b} \eta^{a b}
$$

In this expression $\dot{v}$ and $\dot{w}$ denote the horizontal resp. the vertical velocity components. Thus the original Lagrangian part consisting of the first two terms may be projected down to the base space $B$ yielding $L_{B}=\frac{1}{2} g_{B}(\dot{x}, \dot{x})-V(x)$, where the metric $g_{B}$ on $B$ is defined by the original metric $g$ acting on some horizontal lift of $\dot{x}$ on $B$. As for the last quadratic Casimir-expression (cf. Refs. 6 and 10 it also is a function on the base $B$, since $\eta^{a b}$ is $G$-invariant. The $K$-linear term depending on the vertical fibre velocity $\dot{w}$ via the connection 1 -form $\omega(10)$ is gauge-dependent.

Let us calculate the change of this additional term under an $S_{K}$-transformation. Let $u \mapsto u s$ be an $S_{K}$-transformation, which can be written as $s=e^{\theta^{r} T_{r}} e^{\xi^{p} T_{p}}$ with the help of bases $\left\{T_{r}\right\}$ and $\left\{T_{p}\right\}$ of $t$ and $c$ in the orthogonal decomposition $s_{K}=t \oplus c$. Taking into account the defining properties of a connection, the gauge change in 
$L_{\text {tot }}$ is calculated to be a total time derivative only,

$$
\Delta L_{\mathrm{tot}}=\Delta L_{K}=\frac{d}{d t} \operatorname{Tr}(K \theta)
$$

which implies the $S_{K}$-symmetry at the classical level. The quantum $S_{K}$-symmetry of the theory on the other hand requires the path-integral (21) to be indepent of the gauge fixing. This is the case if and only if paths related to each other by a gauge transformation contribute to the path-integral with the same amplitude. This requirement is stronger than the classical one in (23) and leads to a quantization of $K$ : consider a path $u(t), t \in[0, T]$, in (21). Gauge related paths contributing to the transition from $u(0)$ to $u(T)$ are given by

$$
u_{s}(t)=u(t) s(t), \quad s(t) \in S_{K}, \quad s(0)=s(T)=1 .
$$

The amplitude of $u_{s}(t)$ differs from that of $u(t)$ by an phase given by

$$
\Delta=\frac{1}{\hbar} \int_{0}^{T} d t \Delta L_{\mathrm{tot}}=\frac{1}{\hbar} \operatorname{Tr}(K(\theta(T)-\theta(0)))
$$

The $S_{K}$-symmetry thus requires $\Delta \in 2 \pi \mathbf{Z}$. This together with the periodicity property of $S_{K}, \theta^{r}(T)-\theta^{r}(0) \in 2 \pi \mathbf{Z}$ implies that each component $K^{r}$ of $K$ must be quantized in multiples of $\hbar$, labelling the inequivalent quantizations. ${ }^{5}$

As mentioned already in the introduction, note that, since the components $K^{r}$ of $K$ are discrete multiples of $\hbar,{ }^{14}$ they all become zero in the classical limit. Thus, although the inequivalent quantum systems have been obtained by quantizing different classical systems, which correspond to different foliations of the Marsden-Weinstein reduction, ${ }^{11}$ according to our philosophy explained in the introduction we view these quantum systems as inequivalent quantizations of the same classical system to which all these quantum sectors reduce in the classical limit. 
Inequivalent quantizations considered so far are of physical interest when quantizing a classical system on a given topologically non-trivial configuration space $B$. One seeks for a principal bundle structure $P$ with a total space tractable for quantization. For example if the base space is not simply connected, one can try first a quantization on its universal covering. If this works, then, besides the "canonical" quantization on $B$, we automatically obtain a series of inequivalent quantum systems. These may be considered to be a result of the non-trivial topology of the base space.

The quantization method discussed so far however suffers from an arbitrariness of the geometry chosen on $P$, especially the form of the metric employed. Thus in order the above procedure to be of real interest the geometric structure on $P$ must be such that it arises naturally from the mathematical or physical setting. For example in case of a Lie group $G$ over the homogeneous space $G / H$ as considered in Refs. 5 and 6 the metric on $G$ is given by an $\operatorname{ad}_{G}$-invariant one. In what follows we shall consider gauge theories. In this case the base configuration space, which is the quotient of the affine space of connection forms and the gauge transformation group, has a complex topology in contrast to the affine total space, on which a natural metric can be chosen.

\section{$3 \quad$ Yang-Mills system}

In this section we apply the inequivalent quantizations method to gauge theory. For simplicity we take the structure group $G$ to be $\mathrm{SU}(N)$. As is well known the action of the gauge group makes the space of connections a principal bundle. ${ }^{20,21}$ More precisely, in this paper we consider gauge potentials $A_{\mu}$ on $\mathbf{R} \times \Sigma$, where $\mathbf{R}$ denotes 
the time coordinate and $\Sigma$ is the 3 -sphere. This topology of spacetime may arise e.g. if gauge fields defined on the Minkowski spacetime $\mathbf{R}^{4}$ fall off at space-like infinity fast enough so that they can be considered as fields on $\mathbf{R} \times \Sigma$. Also, in the following discussion more general cases where $\Sigma$ is an arbitrary closed 3-manifold may be treated analogously. Let $P$ be a principal $G$-bundle and $\mathcal{C}:=\left\{A=A_{i} d x^{i} \mid i=1,2,3\right\}$ the space of irreducible space-component connection 1-forms. Let $\mathcal{G}$ denote the gauge group of all time-independent gauge transformations, which are sections into the adjoint group bundle defined by $P \times_{\text {Ad }} G$. Then the action of $\mathcal{G}$ on $\mathcal{C}$ makes it a principal $\mathcal{G}$-bundle over the orbit space. ${ }^{21}$ The space $\mathcal{C}$ is an affine space and has a natural metric determined by the space components of the spacetime metric and the Killing metric of the structure group: Let ad $P$ be the adjoint vector bundle defined by $P \times_{\text {ad }} \mathcal{L}(G)$, where the structure group $G$ acts on its Lie algebra $\mathcal{L}(G)$ by the adjoint representation. Then the canonical non-degenerate positive metric is defined on the space $\Gamma^{m}$ of $\operatorname{ad} P$-valued $m$-forms, $\Gamma^{m}=\Lambda^{m}(\Sigma) \otimes \operatorname{ad} P$, by

$$
(\alpha, \beta)=\int_{\Sigma} \operatorname{tr}\left(\alpha \wedge^{*} \beta\right)=\int_{\Sigma} \frac{1}{m !} \alpha_{\mu_{1} \cdots \mu_{m}}^{a} \beta_{a}^{\mu_{1} \cdots \mu_{m}} \sqrt{g} d^{3} x
$$

where $*$ denotes the Hodge operator and $g$ stands for the determinant of the metric. Since the tangent space at any point in $\mathcal{C}$ is the space $\Gamma^{1}$ of ad $P$-valued 1 -forms, eq. (26) defines a canonical gauge-invariant metric on $\mathcal{C}$ and thus determines a welldefined connection on $\mathcal{C},{ }^{20}$ see below. Note that $\Gamma^{0}$ of sections of $\operatorname{ad} P$ is the Lie algebra of the gauge group $\mathcal{G}$ (see e.g. Ref. 22 ), which we denote by $\mathcal{L}(\mathcal{G})$. It is equipped with the ad-invariant metric (26).

The covariant derivative $\nabla: \Gamma^{m} \rightarrow \Gamma^{m+1}$ and its adjoint $\nabla^{*}: \Gamma^{m} \rightarrow \Gamma^{m-1}$ with respect to the metric (26) are given by

$$
\nabla \alpha=d \alpha+[A, \alpha], \quad\left(\nabla^{*} \alpha, \beta\right):=(\alpha, \nabla \beta)
$$


where $\alpha \in \Gamma^{m}$ and $\beta \in \Gamma^{m-1}$.

Let us now turn to the standard formulation of Yang-Mills theory itself. The curvature and the action are given by

$$
\begin{aligned}
F_{\mu \nu} & =\partial_{\mu} A_{\nu}-\partial_{\nu} A_{\mu}+\left[A_{\mu}, A_{\nu}\right] \\
I & =\int d t L:=\int d t \int_{\Sigma}\left(\sqrt{g} d^{3} x\right) \frac{1}{4} \operatorname{tr}\left(F_{\mu \nu} F^{\mu \nu}\right) .
\end{aligned}
$$

In the framework of canonical formalism ${ }^{1,20}$ we make use of gauge invariance and set $A_{0}=0$. The coordinate variables are the space components of the potential. Denoting the partial time derivative by a dot, the electric and magnetic field strength components read

$$
\begin{aligned}
E & =F_{i}^{0} d x^{i}=\dot{A}, \\
B & =\frac{1}{2} \epsilon_{i j k} F^{j k} d x^{i},
\end{aligned}
$$

where $A=A_{i} d x^{i}$. The canonical momenta are given by

$$
\pi=\pi_{i}^{a} T_{a} d x^{i}=\frac{\delta L}{\delta \partial_{t} A_{a}^{i}} T_{a} d x^{i}=\dot{A}
$$

and the Hamiltonian by

$$
H=\frac{1}{2}(\pi, \pi)+\frac{1}{2}(B, B) .
$$

As in the case of a point particle on a principal bundle discussed in the previous section the momentum $\pi$ is defined on the tangent space. The supplementary Gauss conditions

$$
\nabla^{*} \pi=0
$$

express the gauge invariance of the Yang-Mills theory and constrain it from the whole connection space $\mathcal{C}$ to the gauge orbit space. To show that the Gauss laws exactly 
correspond to the constraints $(13)^{15,16}$ and moreover that the whole geometrical settings match those of the previous section let us have a closer look at the differential geometric structure of the $\mathcal{G}$-principal bundle $\mathcal{C} .^{20,23,22,24,25}$ Consider an infinitesimal gauge transformation $g=e^{\theta}$ of the potential

$$
A \mapsto A^{g}:=g^{-1} A g+g^{-1} d g \approx A+\nabla \theta
$$

This can be interpreted as follows: The expression $\nabla \theta$ is an element of $\Gamma^{1}$ and is the tangent vector in $T_{A} \mathcal{C}$ generating the gauge transformation on $\mathcal{C}$. In fact it is the fundamental vector field related to $\theta$, which is an element of $\Gamma^{0}$ equivalent to the Lie algebra $\mathcal{L}(\mathcal{G})$. Thus the image of the covariant derivative $\nabla$ in the tangent space at a point $A \in \mathcal{C}$ is given by the vertical tangent vectors along the gauge fibres of $\mathcal{C}$ considered as $\mathcal{G}$-principal bundle, which we denote by $V_{A} \mathcal{C}$. To construct its orthogonal complement, let $\square:=\nabla^{*} \nabla$ be the covariant Laplacian acting on $\Gamma^{1}$ and let $\mathrm{G}=\square^{-1}$. To be precise, this inverse is defined if the connection is irreducible, the set of which however is known to be dense in $\mathcal{C} .{ }^{25}$ The vertical projection is then defined by

$$
\Pi:=\nabla \mathrm{G} \nabla^{*}
$$

and the horizontal component of $T_{A} \mathcal{C}$ is given by $H_{A} \mathcal{C}:=(1-\Pi) T_{A} \mathcal{C}$. The corresponding connection $\omega$ of the orthogonal decomposition $T_{A} \mathcal{C}=H_{A} \mathcal{C} \oplus V_{A} \mathcal{C}$ reads ${ }^{24}$

$$
\begin{aligned}
\omega: T_{A} \mathcal{C} & \longrightarrow \mathcal{L}(\mathcal{G}) \\
\tau & \longmapsto \mathrm{G} \nabla^{*} \tau .
\end{aligned}
$$

It is easy to check that $\omega$ so defined is indeed a connection.

By analogy with $(\overline{7})$ the momentum functional reads $(\theta \in \mathcal{L}(\mathcal{G}))$

$$
J(\theta)(\pi)=(\pi, \nabla \theta)=\left(\nabla^{*} \pi, \theta\right)
$$


So the momentum is linked to the connection via $\square$, which plays the role of what was called $\eta$ in (12). The constraints corresponding to (13), when written without components, are exactly the Gauss laws (34). In order to apply the method of inequivalent quantizations, we refer to the modifications of the constraints (15) in the last section and thus modify the Gauss laws as

$$
\nabla^{*} \pi-K=0
$$

with field-independent $K \in \mathcal{L}(\mathcal{G})$. As has been indicated by (16) for the finite dimensional case, also in Yang-Mills system there are more than one modification procedure possible. As an example, the one paralleling with (16) would read

$$
\nabla^{*} \pi-\square K=0
$$

This constraint shift is field-dependent, and its Poisson algebra becomes rather untractable, contrary to that of (40), which is straightforward. We therefore modify the Gauss constraints by means of (40). This form of modification can be obtained also by simple guess, but we must hold in mind that it is a very special case among possible other modifications.

Employing the standard canonical symplectic structure, we find that the Poisson commutation relation for these modified constraints reads now

$$
\left\{\left(u, \nabla^{*} \pi-K\right),\left(v, \nabla^{*} \pi-K\right)\right\}=\left([u, v], \nabla^{*} \pi-K\right)+([u, v], K) .
$$

The last term in this equation spoiling the closedness of the algebra vanishes whenever $u$ or $v$ are sections in the kernel bundle $\operatorname{Ker}\left(\operatorname{ad}_{K}\right)$. For simplicity of the following discussions let us assume that $K(x, t)$ is a regular semisimple element for 
all $x \in \Sigma$ (so that the kernel bundle is indeed well-defined) and, furthermore, that the kernel bundle is trivial. We may then take normalized base vector fields $\sigma_{s}$, $s=1, \ldots, \operatorname{dim}\left(s_{K}\right)$, and obtain the components of the first class constraints as

$$
\left(\nabla^{*} \pi, \sigma_{s}\right)-\left(K, \sigma_{s}\right)=0 \Leftrightarrow\left(\nabla^{*} \pi\right)_{s}-K_{s}=0
$$

Since $K$ does not depend on fields $A$ and $\pi$, all components of the constraints (40), the first class as well as the second class ones, commute exactly with the Hamiltonian and thus, if $K$ is assumed to be time-independent, $\frac{\partial}{\partial t} K=0$, all constraints are preserved in time. Since the extra term $([u, v], K)$ in (42) is field-independent, there are no additional second order constraints. Due to the presence of second class constraints in (40) the full gauge symmetry is lost and there is only a residual $S_{K^{-}}$ symmetry.

We now consider the quantization of the modified Yang-Mills system at hand by means of path-integral method. As described in the previous section we introduce gauge fixings for the $S_{K}$-symmetry and include all the constraints into the phase space path-integral analogously to (20). The delta functionals for the first class constraints (43) are implemented in a standard way into the phase factor by using the time component of the connection $A_{0} \cdot{ }^{1}$ The background of the calculational steps in the path-integral based on the canonical theory of Yang-Mills system have been explained in detail in the review by Jackiw ${ }^{1}$ and will not be mentioned here. Performing the $\pi$-integration one is finally led to a modified Lagrangian

$$
\begin{aligned}
L_{\mathrm{tot}} & =L+L_{K} \\
& =L+\left(A_{0}, K\right) .
\end{aligned}
$$

Contrary to the finite-dimensional case (22) the Casimir-term is missing in (44). This is due to the fact that the delta functionals have not been evaluated completely as 
in the previous section but only implemented into the Lagrangian by employing the time-component $A_{0}$ as Lagrange multiplier. Thus the $K$-quadratic term is implicitly contained in the Lagrangian, which becomes explicit when the field equation $\nabla^{*} E=K$ is taken into account. The time-component is used to maintain Lorentz covariance, see also below.

Under an infinitesimal $S_{K}$-gauge transformation $g=e^{\theta}$, where $[\theta, K]=0$, the Lagrangian changes by a total time derivative only, cf. eq. (23)

$$
\Delta L_{\mathrm{tot}}=\left(\dot{\theta}+\left[A_{0}, \theta\right], K\right)=\frac{d}{d t}(\theta, K) .
$$

The quantization conditions of the component fields $K_{s}$ may be obtained exactly in the same way as described in (24) and (25) and yields

$$
\frac{1}{\hbar}\left(\theta_{s}(T)-\theta_{s}(0), K\right)=\frac{2 \pi n}{\hbar} \int_{\Sigma} K_{s} \in 2 \pi \mathbf{Z}, \quad n \in \mathbf{Z},
$$

where $\theta_{s}(x, t), t \in[0, T]$, is a time-dependent $S_{K}$-gauge transformation with the boundary conditions $e^{\theta_{s}(0)} \equiv e^{\theta_{s}(T)} \equiv 1$. In view of the equations of motion, $\nabla^{*} E=$ $K$, we conclude that the charges associated with the Cartan subgroup of $G$ are quantized,

$$
Q_{s}:=\int_{\Sigma}\left(\nabla^{*} E\right)_{s} \in \hbar \cdot \mathbf{Z}
$$

and, owing to the abelian nature of the residual Cartan subgroup, these external charges are covariantly conserved and static. As in the introduction and also in the previous section we note that, since these external charges $Q_{s}$ in (47) are multiples of $\hbar$, all the inequivalent quantum sectors of the Yang-Mills system reduce to the distinguished classical system described by the usual Gauss law (34) in the classical $\operatorname{limit} \hbar \longrightarrow 0$.

In (44) it is not apparent that $L_{K}$ induces a topological charge. This is so because the Lorentz covariance was lost in applying the canonical formalism. However, we 
may recover the Lorentz covariance in the following way. We first look at the action of the $K$-linear Lagrangian part in the time interval $[0, T]$ under consideration,

$$
I_{K}=\int_{0}^{T} L_{K} d t=\int_{[0, T] \times \Sigma} A_{0}^{a} K_{a} d V
$$

where $d V:=\sqrt{g} d^{3} x d t$ is the 4 -volume form. Since the entire expression in the spacetime integral is Lorentz invariant and $A_{0}^{a}$ is the 0-component of a 4 -form, $K_{a}$ itself must be the 0-component of some $K_{a}^{\mu}$, say. Also, the requirement of time independence of $K_{a}$ stated before has as its covariant counterpart the vanishing of the divergence $\partial_{\mu}\left(K_{a}^{\mu} \sqrt{g}\right)$. Using the language of differential forms, we may express the integrand in (48) as $i_{K} A \wedge d V$, where $i_{K}$ denotes the interior product of forms and vectors. This expression is equivalent to $A \wedge i_{K} d V$, and the 3 -form $\tilde{K}:=i_{K} d V$ so introduced is exact. The phase difference $\Delta W_{K}$ induced by the above mentioned $S_{K}$-gauge transformation may be derived, using $\partial \Sigma=\emptyset$, as

$$
\begin{aligned}
\Delta I_{K} & =\int_{[0, T] \times \Sigma} \operatorname{tr} \nabla \theta \wedge \tilde{K}=\int_{[0, T] \times \Sigma} \operatorname{tr} d \theta \wedge \tilde{K} \\
& =\int_{\partial([0, T] \times \Sigma)} \operatorname{tr} \theta \tilde{K} \\
& =\int_{\Sigma} \operatorname{tr}(\theta(T)-\theta(0)) \tilde{K} .
\end{aligned}
$$

The quantum condition (46) now reads

$$
Q_{s}=\int_{\Sigma}\left(\nabla^{*} E\right)_{s}=\int_{\Sigma} \tilde{K}_{s} \in \hbar \cdot \mathbf{Z}
$$

where the exact forms $\tilde{K}_{s}$ represent elements in the de Rham cohomology group, $\left[\tilde{K}_{s}\right] \in \mathrm{H}^{3}(\Sigma ; \mathbf{R})$. In this way the charges associated with the residual Cartan subgroup are linked to the topology of the underlying space $\Sigma$. If gauge-coupled fermionic matter is included, the whole quantization procedure goes through without changes, and on the lefthand side of (50) matter charge adds up. Equation (50) 
also states the absence of any superselection sector if the topology of the space is trivial, i.e. $\mathrm{H}^{3}(\Sigma ; \mathbf{R})=0$.

\section{Discussion and Outlook}

In the previous section we have employed the method of inequivalent quantizations in the simplest fashion, where a field-independent constraint shift was chosen. As a result the Yang-Mills quantum system contains superselection sectors labelled by quantized external charges arising from the non-trivial topology of the underlying space.

These quantized charges have nothing to do with the topological charge (Pontryagin index) of instantons, since in our case the residual Cartan symmetry is abelian. Contrary to those topological charges, which directly give insight into the topology of the configuration space $\mathcal{C} / \mathcal{G}$ in that they label the connected components, the inequivalent quantizations induced by the simple modification (40) of the Gauss constraints could only detect the topology of the underlying space $\Sigma$. However, the idea of inequivalent quantizations as explained especially in Ref. 5 can incorporate more general quantum constraints than those induced by the modified Gauss laws (40) corresponding to Marsden-Weinstein reductions. ${ }^{13}$ With a more systematic analysis of other field-dependent modifications like (41) it may be possible to unveil the quantum implications of the topology of configuration space itself. Especially, one should look for induced Lagrangian parts which are explicitly topological, since then we can be fairly sure that they are closely linked to the topology of the gauge orbit space, as can be seen from the work of $\mathrm{Wu}$ and Zee. ${ }^{2}$

Another remaining issue is the investigation of the induced connection ${ }^{10,5}$ on the 
gauge orbit space. For example, the one induced by the above choice (40) is seen to be non-exact in the functional sense, and one might wonder whether it is possible to relate these induced connections to the Chern classes of the orbit space, which is known to be a classifying space for the gauge group $\mathcal{G}$.

Acknowledgements: I would like to greatly thank Prof. Izumi Tsutsui for not only suggesting this line of research but also for his many helpful discussions; moreover, I am very much indebted to Prof. Martin Kretzschmar, who helped to resolve an organizational problem during the preparation of this work. 


\section{References}

1. R. Jackiw, Rev. Mod. Phys. 52, 661 (1980).

2. Y.-S. Wu, A. Zee, Nucl. Phys. B258, 157 (1985).

3. L. Alvarez-Gaumé, P. Ginsparg, Nucl. Phys. B 243, 449 (1984).

4. A. Heil, A. Kersch, N. Papadopoulos, B. Reifenhäuser, F. Scheck, J. Geom. Phys. 7, 489 (1990).

5. D. McMullan, I. Tsutsui, Ann. Phys. (N.Y.) 237, 269 (1995).

6. T. Tanimura, I. Tsutsui, Ann. Phys. (N.Y.) 258, 137 (1997).

7. G. W. Mackey, Induced Representation of Groups and Quantum Mechanics (Benjamin, New York, 1969).

8. M. G. G. Laidlaw, C. M. DeWitt, Phys. Rev. D3, 1375 (1971).

9. C. J. Isham, in Relativity, Groups and Topology II eds. B. S. DeWitt and R. Stora (North-Holland, Amsterdam, 1984).

10. N. P. Landsman, N. Linden, Nucl. Phys. B 365, 121 (1991).

11. M. A. Robson, J. Geom. Phys. 19, 207 (1996).

12. P. A. M. Dirac, Lectures on Quantum Mechanics (Yeshiva, New York, 1964).

13. V. Guillemin, S. Sternberg, Symplectic techniques in physics (Cambridge University Press, Cambridge, 1984).

14. D. McMullan, I. Tsutsui, Phys. Lett. B. 320, 287 (1994). 
15. J. M. Arms, Math. Proc. Camb. Phil. Soc. 90, 361 (1981).

16. J. M. Arms, Acta Phys. Polon. B17, 499 (1986).

17. J. E. Marsden, Lectures on Mechanics (Cambridge Univ. Press, Cambridge, 1992).

18. R. Montgomery, Lett. Math. Phys. 8, 59 (1984).

19. R. Abraham, J. E. Marsden, Foundations of Mechanics (Addison-Wesley, New York, 1978), 2nd edition.

20. O. Babelon, C. M. Viallet, Commun. Math. Phys. 81, 515 (1981).

21. M. S. Narasimhan, T. R. Ramadas, Commun. Math. Phys. 67, 121 (1979).

22. P. K. Mitter, C. M. Viallet, Commun. Math. Phys. 79, 457 (1981).

23. M. Daniel, C. M. Viallet, Rev. Mod. Phys. 52, 175 (1980).

24. C.-M. Viallet, in Physics, Geometry, and Topology, eds. H. C. Lee (Plenum Press, New York, 1990).

25. I. M. Singer, Comm. Math. Phys. 60, 7 (1978). 